\title{
Contrast-Enhanced MRI Texture Parameters as Potential Prognostic Factors for Primary Central Nervous System Lymphoma Patients Receiving High-Dose Methotrexate-Based Chemotherapy
}

\author{
Chaoyue Chen $\mathbb{D},{ }^{1,2}$ Hongyu Zhuo, ${ }^{1,3}$ Xiawei Wei $\mathbb{C}^{1},{ }^{1}$ and Xuelei Ma $\mathbb{D}^{1,3}$ \\ ${ }^{1}$ State Key Laboratory of Biotherapy and Cancer Center, West China Hospital, \\ Sichuan University and Collaborative Innovation Center for Biotherapy, Chengdu, China \\ ${ }^{2}$ Department of Neurosurgery, West China Hospital, Sichuan University, Chengdu, China \\ ${ }^{3}$ Department of Biotherapy, Cancer Center, West China Hospital, Sichuan University, Chengdu, China \\ Correspondence should be addressed to Xiawei Wei; xiaweiwei@scu.edu.cn and Xuelei Ma; drmaxuelei@gmail.com
}

Received 19 May 2019; Accepted 26 August 2019; Published 12 November 2019

Academic Editor: Guillermina Ferro-Flores

Copyright (C) 2019 Chaoyue Chen et al. This is an open access article distributed under the Creative Commons Attribution License, which permits unrestricted use, distribution, and reproduction in any medium, provided the original work is properly cited.

Introduction. The purpose of this study was to evaluate the prognostic value of texture features on contrast-enhanced magnetic resonance imaging (MRI) for patients with primary central nervous system lymphoma (PCNSL). Methods. In this retrospective study, fifty-two patients diagnosed with PCNSL were enrolled from October 2010 to March 2017. The texture feature of tumor tissue on the histogram-based matrix (histo-) and the grey-level co-occurrence matrix (GLCM) was retrieved by contrastenhanced T1-weighted imaging before any antitumor treatment. Receiver operating characteristic curve analyses were performed to obtain their optimal cutoff values, based on which we dichotomized patients into subgroups. The Kaplan-Meier analyses were conducted to compare overall survival (OS) of subgroups, and multivariate Cox regression analyses were used to determine if they could be taken as independent prognostic factors. Results. Ten texture features were extracted from the MR image, including Energy, Entropy, Kurtosis, Skewness on the histogram-based matrix, and Correlation, Contrast, Dissimilarity, Energy, Entropy, and Homogeneity on the grey-level co-occurrence matrix. Three of them (GLCM-Contrast, GLCM-Dissimilarity, and GLCMHomogeneity) are shown to be significant in relation to overall survival (OS). The multivariate Cox regression analyses suggest that GLCM-Homogeneity could be taken as independent predictors. Conclusions. The texture features of contrast-enhanced magnetic resonance imaging (MRI) could potentially serve as prognostic biomarkers for PCNSL patients.

\section{Background}

Primary central nervous system lymphoma (PCNSL) is an uncommon type of tumor, accounting for about $1 \%$ nonHodgkin's lymphomas (NHLs) and 3\% primary cerebral tumors. Characterized as a type of aggressive brain tumor, the lymphoma tissues confine to the central nervous system (CNS), including the brain, spinal cord, leptomeninges, and eyes $[1,2]$. The diffuse large B-cell lymphoma (DLBCL) constitutes nearly $95 \%$ of PCNSL while $<4 \%$ of PCNSL are of $\mathrm{T}$-cell origin $[3,4]$. Chemotherapy is the basic treatment for PCNSL, and high-dose methotrexate- (HD-MTX-) based chemotherapy is highly recommended due to the ability in significantly increasing the patient's overall survival time according to recent studies [5, 6]. With the advance in treatment over the last 20 years, the five-year survival rate for patients treated with HD-MTX is approximately 15\% 20\%, especially for those at young age or with tumor tissue with good performance status.

Texture analysis (TA) is a statistical method of analyzing the region of interest (ROI) of an image from plain radiographs, magnetic resonance imaging (MRI), and computed tomography (CT) [7]. Based on the mathematical calculation on spatial organization and distribution of grey 
values, the TA could provide the nonvisual information to assist in diagnosis and to predict the prognosis. Recent studies illustrated that the MRI texture features could be potential prognostic biomarkers for patients with various types of cancer, including breast cancer, rectal cancer, and soft-tissue sarcomas [8-10]. Inspired from these studies, we performed this research to investigate the relationship between MRI texture features and the survival of patients with PCNSL, and to determine if MRI texture features could be reliable parameters in predicting patient survival.

\section{Method}

2.1. Patient Selection. The patients involved in this study were selected from the neurosurgery department of our institution, who were diagnosed and treated between October 2010 and March 2014. Initially, we reviewed all the electronic medical records to evaluate if the potential patients were potentially qualified for this study, and to collect clinical information which could have an impact on patient survival. DLBCL is the most common lymphoma of pathological subtype, bringing the dilemma of scarce information on patients with other lymphoma subtypes and unconvincing statistical analysis on pathological subtype. So we decided to include only DLBCL patients in this study while patients with other types of lymphoma were excluded. The inclusive criteria were as follows: patients (a) with pathological confirmation; (b) with elaborate medical record on the progress of diagnosis and treatment; (c) with an available MRI imagine before antitumor treatment. The exclusive criteria included patients: (a) with a history of surgical intervention of intracranial disease; (b) clinical proof on other diseases that could decrease the survival rate significantly (such as infection, coronary disease, and uremia). Based on this strategy, we made a preliminary list and collected follow-up information from the database. Other clinical parameters, such as age, gender, chemotherapy regime, and radiotherapy regime, were also recorded.

It is worth noting that the role of surgery was controversial in the recent years. The dominant perception was that surgery was not recommended except for biopsy because of the mutual view among the neurosurgeons that there is no benefit from resection for patients. In our study, the neurosurgeons agreed with the standpoint and the purpose of resection for patients was only for biopsy. However, some patients undertook subtotal resections (STRs) due to the radiological misdiagnosis of nontypical lymphoma, while pathologically confirmed by intraoperative frozen section diagnosis during operation.

The endpoint of this study was overall survival (OS), which was calculated as the time from the date of histological diagnosis to the date of tumor-related death or the last time access. Based on the results of telephone follow-ups or medical record, we documented their survival condition until Mar 2019. This study was approved by the medical ethics committee of the institution.

2.2. MRI Acquisition. The MRI scan was conducted via a 3 Tesla GE MRI system with an 8-channel phase-array head coil. The subjects were asked to keep their eyes closed, stay relaxed, and reduce movement. The parameters of the contrast-enhanced T1-weighted imaging were: time repetition $=2000 \mathrm{~ms}$, time echo $=30 \mathrm{~ms}$, flip angle $=90^{\circ}$, slice thickness $=5 \mathrm{~mm}$ (no slice gap), field of view = $240 \times 240 \mathrm{~mm}^{2}, 30$ axial slices, and 200 volumes in each run.

2.3. MRI Image Texture Feature Analysis. The MRI texture features were extracted by two certified neurosurgeons using LIFEx software (http://www.lifexsoft.org.) with assistance of certificated nuclear medicine physicians (Figure 1). It is worth noting that all MR images used for texture analysis were performed before any antitumor treatment bringing down the influence of radio or chemical therapy on texture analysis. As per the instruction of software, 3D ROI was extracted by manually drawing along the lesion slice by slice [11]. Based on feedback from surgeons and statistics of texture features, we retrieved $\mathrm{TA}$ on axial T1-weighted imaging after contrast was chosen to perform survival analysis due to rather good description on the lesion location. The Mann-Whitney $U$ test suggested that there was no statistical difference in features extracted by two users (Supplement Table 1). Eventually, textures of imaging were extracted into a dataset for further analysis from two matrixes, including Energy, Entropy, Kurtosis, Skewness on the histogram-based matrix, and Correlation, Contrast, Dissimilarity, Energy, Entropy, Homogeneity on the grey-level co-occurrence matrix. Partial volume effect correction was not performed.

2.4. Statistical Analysis. In this study, we performed our analyses using MedCalc statistics and SPSS statistics version 21 (IBM Corp., Chicago). The optimal cutoff value was determined at the point of the maximal Youden's index with receiver operating characteristic (ROC) analyses [12]. Kaplan-Meier Curve was performed to illustrate the relationship between specific features and patient survival. Moreover, multivariate analyses were conducted with the Cox proportional hazards regression model to determine their value of being independent predictors. The correlations between features were indicated by Pearson Correlation analysis.

\section{Results}

3.1. Patient Selection. A total number of 52 patients were selected from the database to be involved in this study. The mean age of patients was 53.57 years (range, 29 74 years), and the sex ratio was 29:23 (29 males and 23 females). Two different chemotherapy regimens were applied to these patients based on their situation, high-dose MTX-based chemotherapy (HD-MTX+), and R-CHOP+MTX chemotherapy regimen. The whole brain radiotherapy (WBRT) was applied to 11 patients as radiotherapy. The detailed characteristics of patients are summarized in Table 1. One-way ANOVA analysis suggested that the radiotherapy was 

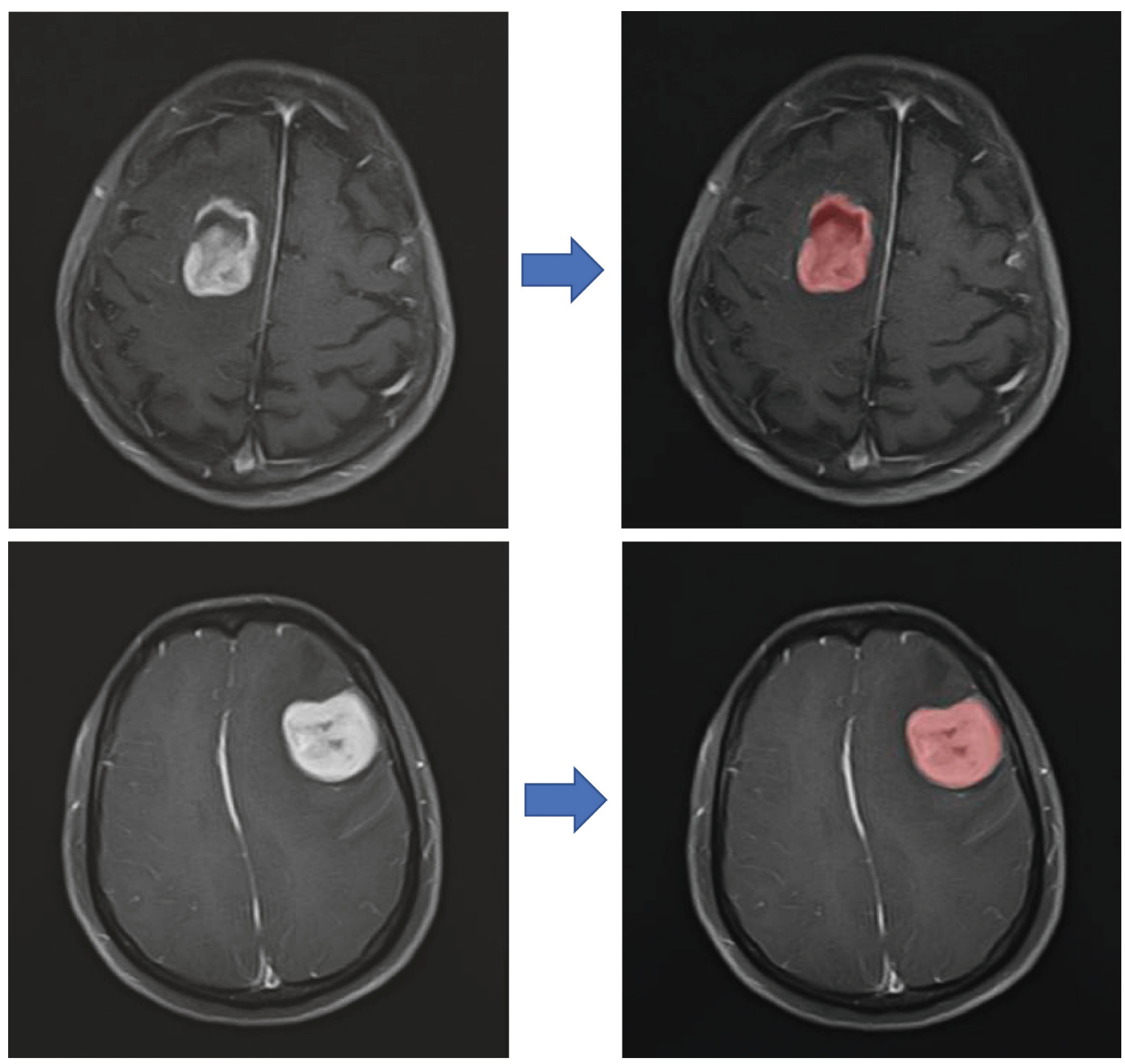

Figure 1: The screenshot of the texture features extraction.

TABLE 1: Characteristics of patients diagnosed with PCNSL.

\begin{tabular}{lc}
\hline Characteristics & Numbers \\
\hline Age value \\
$\quad<60$ years old & 58.45 (range: $32 \sim 75)$ \\
$>60$ years old & $36(69.2 \%)$ \\
Gender & $16(30.8 \%)$ \\
$\quad$ Male & $29(55.8 \%)$ \\
$\quad$ Female & $23(44.2 \%)$ \\
Surgery & $35(57.7 \%)$ \\
$\quad$ Stereotactic biopsy (STB) & $17(13.5 \%)$ \\
Subtotal resections (STRs) & $49(94.2 \%)$ \\
Chemotherapy & $3(5.8 \%)$ \\
$\quad$ High-dose MTX-based chemotherapy regimen & $11(21.2 \%)$ \\
R-CHOP+MTX chemotherapy regimen & $41(78.8 \%)$ \\
Radiotherapy & \\
$\quad$ Yes & 18 (mean time: 22 months) \\
No & 34 \\
Patient survival status & 0.684 \\
Dead & \\
Alive & \\
\hline
\end{tabular}




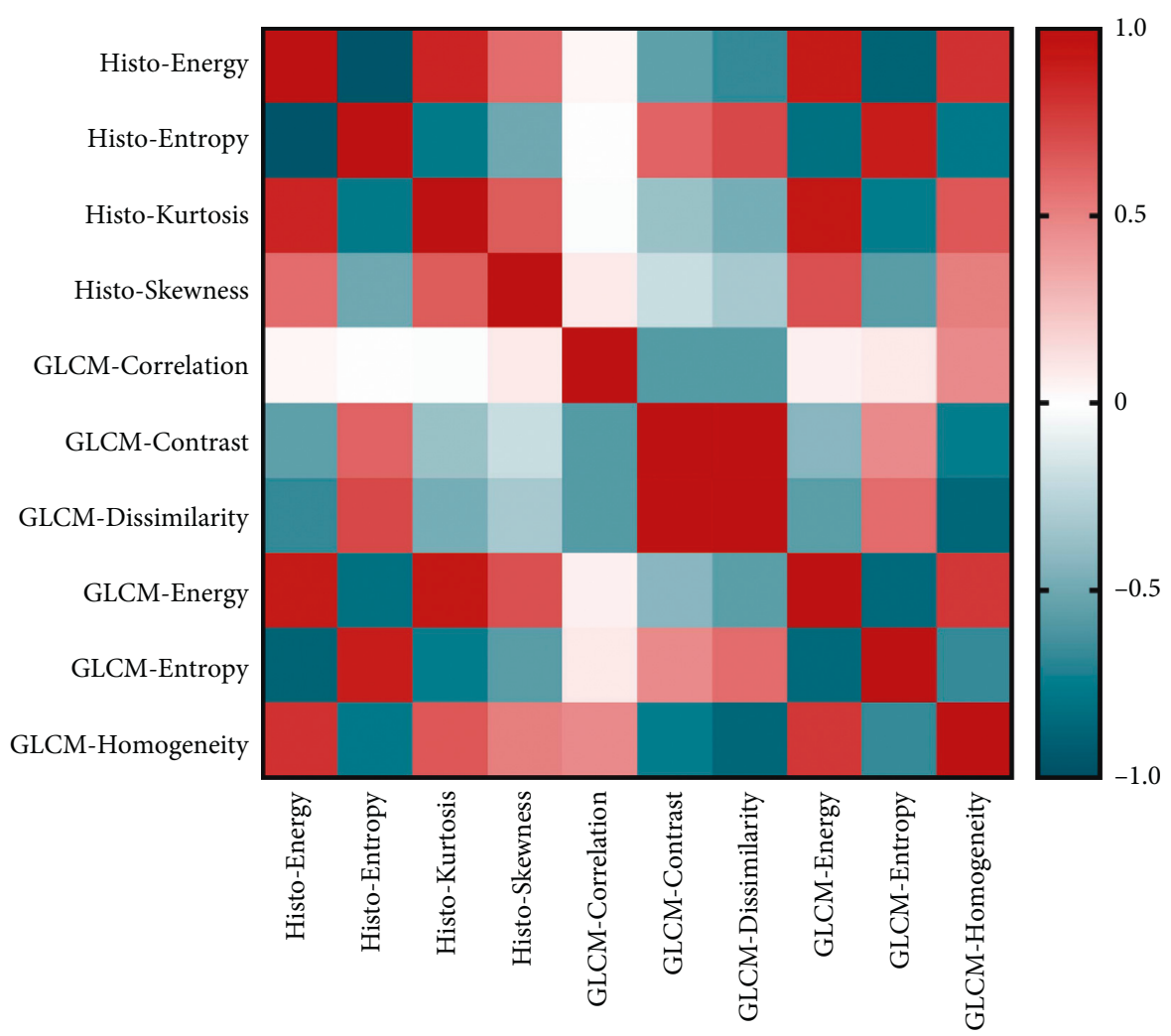

FIGURE 2: HOTMAP of MRI textural parameters.

significantly correlated to patient survival while other clinical parameters were not $(p=0.032)$.

3.2. MRI Texture Features and Survival Analysis. Considering the complicated relationship between texture features, Pearson correlation was conducted first to evaluate the association between features. The result suggested most of the MRI textures were correlated with each other significantly (Figure 2).

The results of ROC analyses showed that 3 of MRI texture features (GLCM-Contrast $(p=0.016)$, GLCMDissimilarity $(p=0.015)$, and GLCM-Homogeneity $(p=$ $0.039)$ ) were found to be significantly related to OS (Table 2). Then we conducted K-M analyses to compare their value to predict OS of patients. The K-M survival analyses demonstrated that the survival rate would be worse in the patients with lower GLCM-Contrast $(p=0.027)$, lower GLCM-Dissimilarity $(p=0.027)$, and higher GLCMHomogeneity $(p=0.014)$ than the optimal cutoff values (Figure 3). Subsequently, multivariate Cox proportional hazards regression analyses were performed to see if they could be considered as independent prognostic parameters. The age of patients and radiotherapy regime were also included into the model considering the impact of age on patient survival. The results showed that GLCM-Homogeneity $(p=0.021, \mathrm{HR}=3.075)$ had potential to be an independent factor while radiotherapy $(p=0.559)$, GLCM-Contrast $(p=0.242)$, and GLCM-Dissimilarity $(p=0.242)$ do not (Table 3$)$.
TABLE 2: Receiver operating characteristic curve analysis on MRI texture features.

\begin{tabular}{lcccc}
\hline $\begin{array}{l}\text { MRI texture } \\
\text { features }\end{array}$ & $\begin{array}{c}\text { ROC } \\
\text { threshold }\end{array}$ & $\begin{array}{c}p \\
\text { value }\end{array}$ & AUC & $95 \%$ CI \\
\hline Histo-Energy & $>0.03$ & 0.179 & 0.615 & $\begin{array}{c}0.470 \text { to } \\
0.747\end{array}$ \\
Histo-Entropy & $\leq 1.54$ & 0.120 & 0.634 & $\begin{array}{c}0.489 \text { to } \\
0.763\end{array}$ \\
Histo-Kurtosis & $>4.07$ & 0.093 & 0.642 & $\begin{array}{c}0.497 \text { to } \\
0.770\end{array}$ \\
Histo-Skewness & $\leq 1.37$ & 0.806 & 0.523 & $\begin{array}{c}0.380 \text { to } \\
\text { ( }\end{array}$ \\
GLCM- & $>0.663$ \\
Correlation & & 0.155 & 0.614 & $\begin{array}{c}0.469 \text { to } \\
0.746\end{array}$ \\
GLCM-Contrast & $<\mathbf{1 2 1 . 1 7}$ & $\mathbf{0 . 0 1 6}$ & $\mathbf{0 . 6 8 1}$ & $\mathbf{0 . 5 3 8}$ to \\
GLCM- & & & & $\mathbf{0 . 8 0 4}$ \\
Dissimilarity & $<7.81$ & $\mathbf{0 . 0 1 5}$ & $\mathbf{0 . 6 8 1}$ & $\mathbf{0 . 5 3 8}$ to \\
GLCM-Entropy & $\leq 2.86$ & 0.163 & 0.614 & 0.469 to \\
& & & & 0.746 \\
GLCM-Energy & $>0.0018$ & 0.087 & 0.636 & $\begin{array}{c}0.491 \text { to } \\
0.765\end{array}$ \\
GLCM- & & & & $\mathbf{0 . 5 1 9}$ to \\
Homogeneity & $\geq \mathbf{0 . 2 9}$ & $\mathbf{0 . 0 3 9}$ & $\mathbf{0 . 6 6 3}$ & $\mathbf{0 . 7 8 8}$ \\
\hline
\end{tabular}

ROC: receiver operating characteristic; AUC: area under curve; CI: confidence interval; GLCM: grey-level co-occurrence matrix.

\section{Discussion}

Our study investigated the relationship between texture features and prognosis of PCNSL patients, suggesting that 


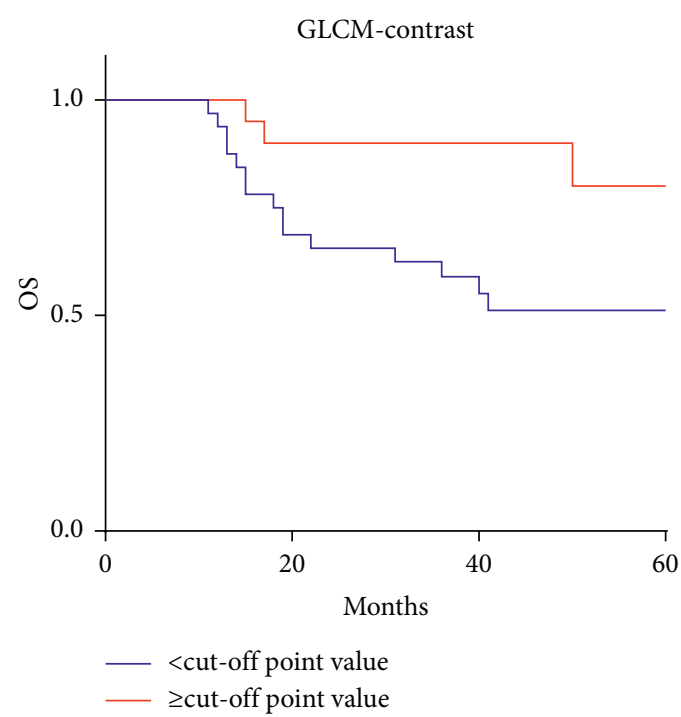

(a)

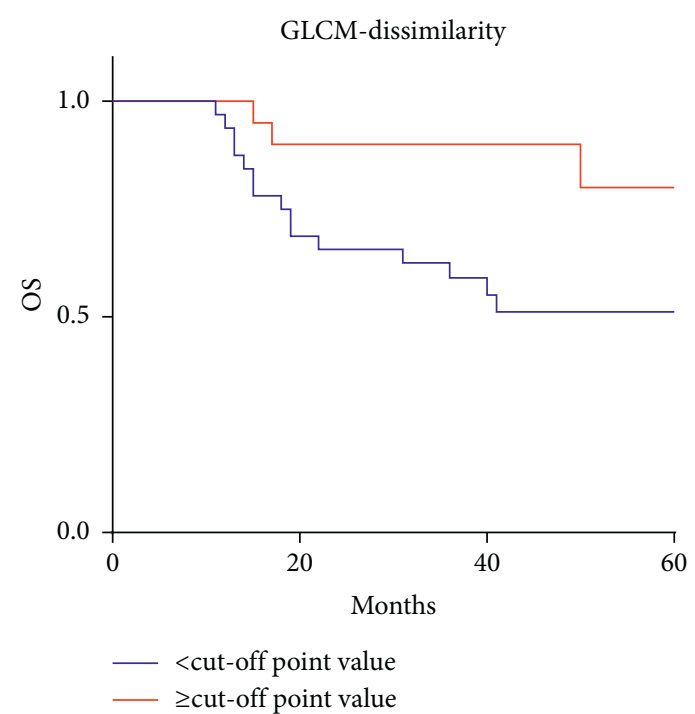

(b)

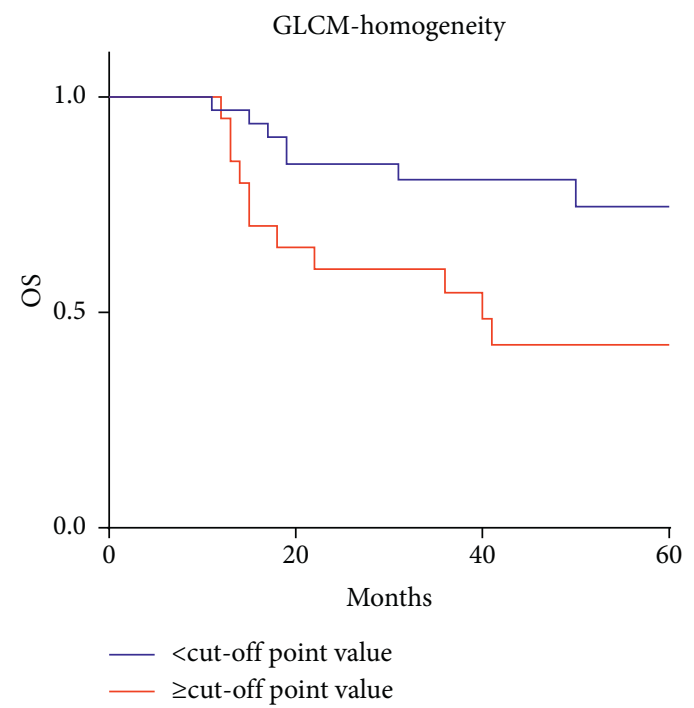

(c)

FIgURE 3: Kaplan-Meier survival curves on the texture parameters of (a) GLCM-Contrast, (b) GLCM-Dissimilarity, and (c) GLCMHomogeneity. Patients with (a) GLCM-Contrast $>121.1726$, (b) GLCM-Dissimilarity $>7.8085$, and (c) GLCM-Homogeneity $<0.2864$ show better survival, with log-rank tests showing $p$ values of $0.027,0.027$, and 0.014 , respectively.

TABLE 3: Multivariate Cox proportional hazard regression analysis on MRI texture parameters.

\begin{tabular}{lccc}
\hline MRI texture features & HR & $p$ value & $95 \%$ CI \\
\hline GLCM-Homogeneity & 3.075 & 0.021 & $1.188 \sim 7.957$ \\
\hline
\end{tabular}

HR: hazard ratio; CI: confidence interval; GLCM: grey-level co-occurrence matrix.

parameters on contrast-enhanced MRI could potentially serve as parameters to predict the prognosis of patients diagnosed with DLBCL, and GLCM-Homogeneity have potential to be a clinically independent predictor.

The prognostic prediction of PCNSL is essential for physicians as they need the information to adapt therapeutic schedule and guide expectations for patients based on their prognosis. Nowadays, the clinicians make prediction on patient survival based on several parameters, including age, Karnofsky performance status (KPS), location of lesions, and blood test results. However, the prognostic value of radiological parameters is still unclear and needed research to verify. In our research, we demonstrated the texture analysis had potential to predict prognosis of PCNSL, technically for patients with DLBCL, hoping to introduce a new set of parameters to assist physicians in making more accurate and precious decisions. To the best of our knowledge, our research is the first one demonstrating the statistically significant association between radiomic on contrast-enhanced MRI and prognosis of PCNSL patients.

Texture analysis was considered as a novel method with great potential and has been developed rapidly in recent years. Given that the MRI is a routine examination for intracranial tumors, TA would provide an efficient method 
to predict patient survival with advantages of convenience and no additional cost. The value of TA in diagnosis and prognosis of intracranial tumors has been indicated before. One study explored the ability of TA in distinguishing SFT/ HPC from meningioma, suggesting that TA could assist surgeons in designing therapeutic and surgical strategy [13]. Another study investigated the diagnostic value of MRI texture features in the discrimination of glioblastoma (GBM) and PCNSL, two of which shared similar visual characteristics on MRI $[14,15]$. The prognostic value of MRI texture has also been illustrated but with relatively limited number. Only one study illustrated the relationship between texture features on MR-perfusion image and survival of PCNSL patients, but negatively association was observed [16]. In our study, the relationship between texture parameters and survival were conducted on contrast-enhanced T1-WI, and the results showed that GLCM-Homogeneity could be considered as an independent prognostic predictor.

The mechanism for TA as prognostic predictors is that texture features are correlated with tumor biology, and the variation on features indicated the tumor progression in this specific malignant lesion. The appearance of PCNSL on MRI is characteristically described as hypercellular and represents homogenous enhancement with or without necrosis after administration of contrast [17]. Theoretically, the characteristics of tumors could be depicted by TA mathematically as spatial frequency, statistical parameters, or structural primitives [18]. The possible explanation for the GLCMHomogeneity as independent predictors was conceptually from the meaning of the feature that it describes the homogeneity of grey-level voxel pairs of images. The variations on parameters might reflect the pathological procedure of tumors such as neovascularization and hypoxia, which eventually result in necrosis represented as in MRI and usually are taken as the sign of poor prognosis.

TA could also have potential to serve as a novel imaging technology in the biology research with the ability in analyzing nonvisual information. The ability of TA on conventional MRI image in discriminating the pathological subtype of intracranial disease has been reported in a previous study [19]. Some researchers have also reported that the profiling of the histogram-based matrix on diffusionweighted MRI (DWI) was able to reflect tumor biological features of PCNSL [20]. And other studies demonstrated that there were significant correlation between the MRIbased textures and tumor pathological process or tumor microenvironment $[19,21]$. However, the consensus has not been reached due to the inadequate evidence, bringing the urgency of more researches in the future to investigate the relationship between specific texture features and tumor characteristics.

There are some limitations in our study. First and foremost, this is a retrospective study with variable time of follow-up periods. A prospective study is required to confirm the reliability of results. Secondly, the population involved in is relatively small even with a large database. The reason is that some patients came from remote areas and were hard to clinically follow in the long-term. Third, some of our results did not accord with previous studies, like the results of univariate analysis which showed that age was negatively significantly related to the patient survival in this study. Even with a similar result from another study, explanation seems not convincing [16]. Fourth, the prognostic value of TA was only applied for the texture features extracted from histo- and GLCM matrixes. The selection bias of 10 parameters used for this study is inevitable. Finally, there are several other pathological subtypes of PCNSL, but only DLBCL were enrolled in this study. The value of texture analysis in other pathological lymphoma subtypes should be verified in future research.

\section{Data Availability}

The data used to support the findings of this study are available from the corresponding author upon request.

\section{Ethical Approval}

All procedures performed in studies involving human participants were in accordance with the ethical standards of the institutional and/or national research committee and with the 1964 Helsinki Declaration and its later amendments or comparable ethical standards. The institutional review board approved this retrospective study.

\section{Consent}

Informed consent was obtained from all individual participants included in the study.

\section{Conflicts of Interest}

The authors declare that they have no conflicts of interest.

\section{Authors' Contributions}

Chaoyue Chen and Xuelei Ma contributed equally to this work.

\section{Supplementary Materials}

Supplement Table 1: Mann-Whitney $U$ test on texture features extracted by two neurosurgeons. (Supplementary Materials)

\section{References}

[1] M. Krogh-Jensen, F. D’Amore, M. K. Jensen et al., "Clinicopathological features, survival and prognostic factors of primary central nervous system lymphomas: trends in incidence of primary central nervous system lymphomas and primary malignant brain tumors in a well-defined geographical area; population-based data from the Danish lymphoma registry, lyfo, and the Danish cancer registry," Leukemia \& Lymphoma, vol. 19, no. 3-4, pp. 223-233, 1995.

[2] K. S. Panageas, E. B. Elkin, L. M. DeAngelis, L. Ben-Porat, and L. E. Abrey, "Trends in survival from primary central nervous system lymphoma, 1975-1999 a population-based analysis," Cancer, vol. 104, no. 11, pp. 2466-2472, 2005.

[3] T. N. Shenkier, J.-Y. Blay, B. P. O’Neill et al., "Primary CNS lymphoma of T-cell origin: a descriptive analysis from the 
international primary CNS lymphoma collaborative group," Journal of Clinical Oncology, vol. 23, no. 10, pp. 2233-2239, 2005.

[4] K. Sinicrope and T. Batchelor, "Primary central nervous system lymphoma," Neurologic Clinics, vol. 36, no. 3, pp. 517-532, 2018.

[5] P. C. O’Brien, D. E. Roos, G. Pratt et al., "Combined-modality therapy for primary central nervous system lymphoma: longterm data from a phase II multicenter study (trans-tasman radiation oncology group)," International Journal of Radiation Oncology Biology Physics, vol. 64, no. 2, pp. 408-413, 2006.

[6] M. Sierra del Rio, A. Rousseau, C. Soussain, D. Ricard, and K. Hoang-Xuan, "Primary CNS lymphoma in immunocompetent patients," The Oncologist, vol. 14, no. 5, pp. 526539, 2009.

[7] A. Kassner and R. E. Thornhill, "Texture analysis: a review of neurologic MR imaging applications," American Journal of Neuroradiology, vol. 31, no. 5, pp. 809-816, 2010.

[8] P. Taourel, E. Pages, I. Millet et al., "Magnetic resonance imaging in breast cancer management in the context of neoadjuvant chemotherapy," Critical Reviews in Oncology/Hematology, vol. 132, pp. 51-65, 2018.

[9] M. Aker, B. Ganeshan, A. Afaq, S. Wan, A. Groves, and T. Arulampalam, "Magnetic resonance texture analysis in identifying complete pathological response to neoadjuvant treatment in locally advanced rectal cancer," Diseases of the Colon \& Rectum, vol. 62, no. 2, pp. 163-170, 2018.

[10] A. Crombe, C. Perier, M. Kind et al., "T2-based MRI deltaradiomics improve response prediction in soft-tissue sarcomas treated by neoadjuvant chemotherapy," Journal of Magnetic Resonance Imaging, vol. 50, no. 2, pp. 497-510, 2018.

[11] C. Nioche, F. Orlhac, S. Boughdad et al., "LIFEx: a freeware for radiomic feature calculation in multimodality imaging to accelerate advances in the characterization of tumor heterogeneity," Cancer Research, vol. 78, no. 16, pp. 4786-4789, 2018.

[12] W. J. Youden, "Index for rating diagnostic tests," Cancer, vol. 3, no. 1, pp. 32-35, 1950.

[13] T. Kanazawa, Y. Minami, M. Jinzaki, M. Toda, K. Yoshida, and H. Sasaki, "Preoperative prediction of solitary fibrous tumor/hemangiopericytoma and angiomatous meningioma using magnetic resonance imaging texture analysis," World Neurosurgery, vol. 120, pp. e1208-e1216, 2018.

[14] D.-D. Xiao, P.-F. Yan, Y.-X. Wang, M. S. Osman, and H.-Y. Zhao, "Glioblastoma and primary central nervous system lymphoma: preoperative differentiation by using MRIbased 3D texture analysis," Clinical Neurology and Neurosurgery, vol. 173, pp. 84-90, 2018.

[15] A. Kunimatsu, N. Kunimatsu, K. Kamiya, T. Watadani, H. Mori, and O. Abe, "Comparison between glioblastoma and primary central nervous system lymphoma using MR imagebased texture analysis," Magnetic Resonance in Medical Sciences, vol. 17, no. 1, pp. 50-57, 2018.

[16] S. Blasel, R. Vorwerk, M. Kiyose et al., "New MR perfusion features in primary central nervous system lymphomas: pattern and prognostic impact," Journal of Neurology, vol. 265, no. 3, pp. 647-658, 2018.

[17] A. Fitzsimmons, K. Upchurch, and T. Batchelor, "Clinical features and diagnosis of primary central nervous system lymphoma," Hematology/Oncology Clinics of North America, vol. 19, no. 4, pp. 689-703, 2005.
[18] G. Castellano, L. Bonilha, L. M. Li, and F. Cendes, "Texture analysis of medical images," Clinical Radiology, vol. 59, no. 12, pp. 1061-1069, 2004.

[19] Y. Zhang, G. R. W. Moore, C. Laule et al., "Pathological correlates of magnetic resonance imaging texture heterogeneity in multiple sclerosis," Annals of Neurology, vol. 74, no. 1, pp. 91-99, 2013.

[20] S. Schob, B. Münch, J. Dieckow et al., "Whole tumor histogram-profiling of diffusion-weighted magnetic resonance images reflects tumorbiological features of primary central nervous system lymphoma," Translational Oncology, vol. 11, no. 2, pp. 504-510, 2018.

[21] E. S. Ko, J. H. Kim, Y. Lim, B. K. Han, E. Y. Cho, and S. J. Nam, "Assessment of invasive breast cancer heterogeneity using whole-tumor magnetic resonance imaging texture analysis: correlations with detailed pathological findings," Medicine, vol. 95, no. 3, Article ID e2453, 2016. 


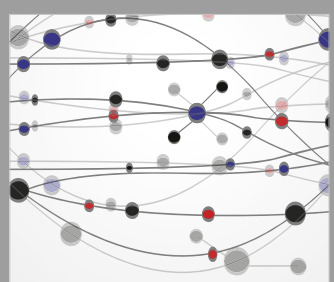

The Scientific World Journal
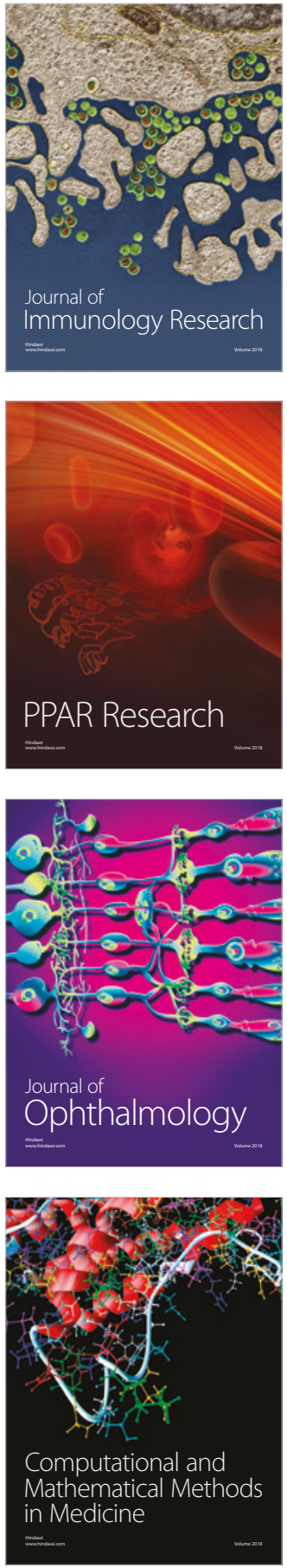

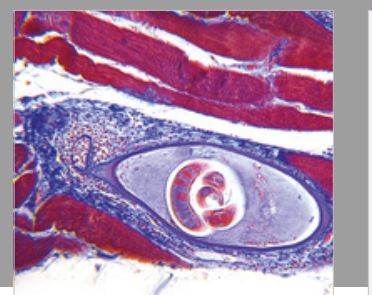

Gastroenterology Research and Practice

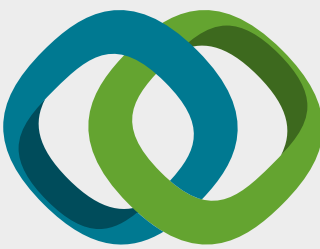

\section{Hindawi}

Submit your manuscripts at

www.hindawi.com
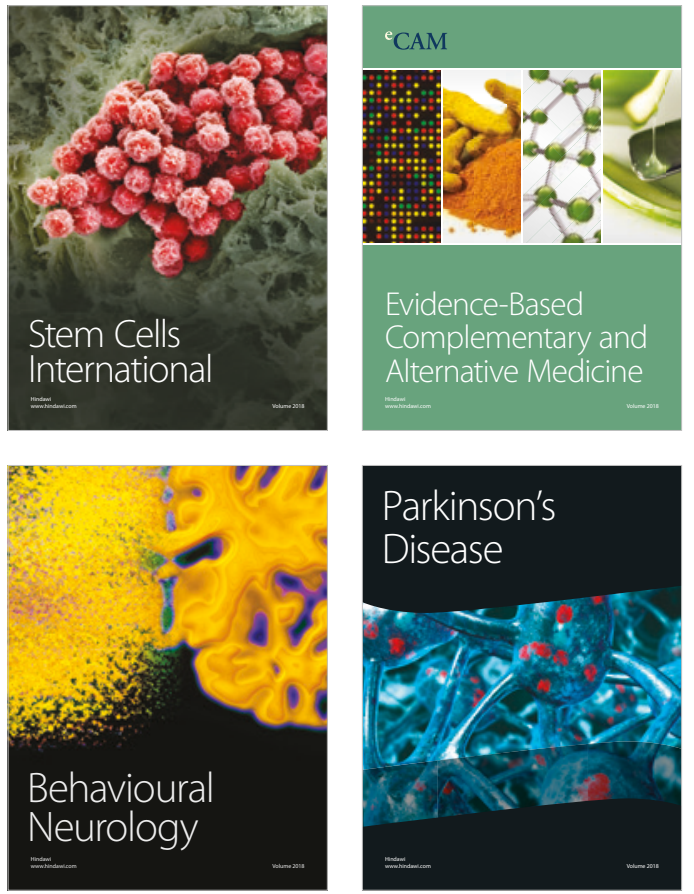

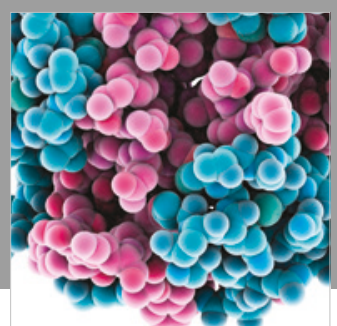

ournal of

Diabetes Research

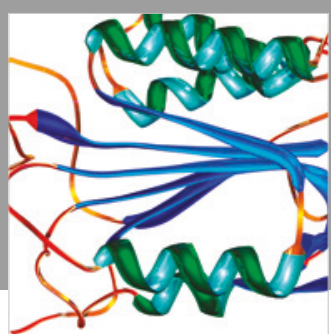

Disease Markers
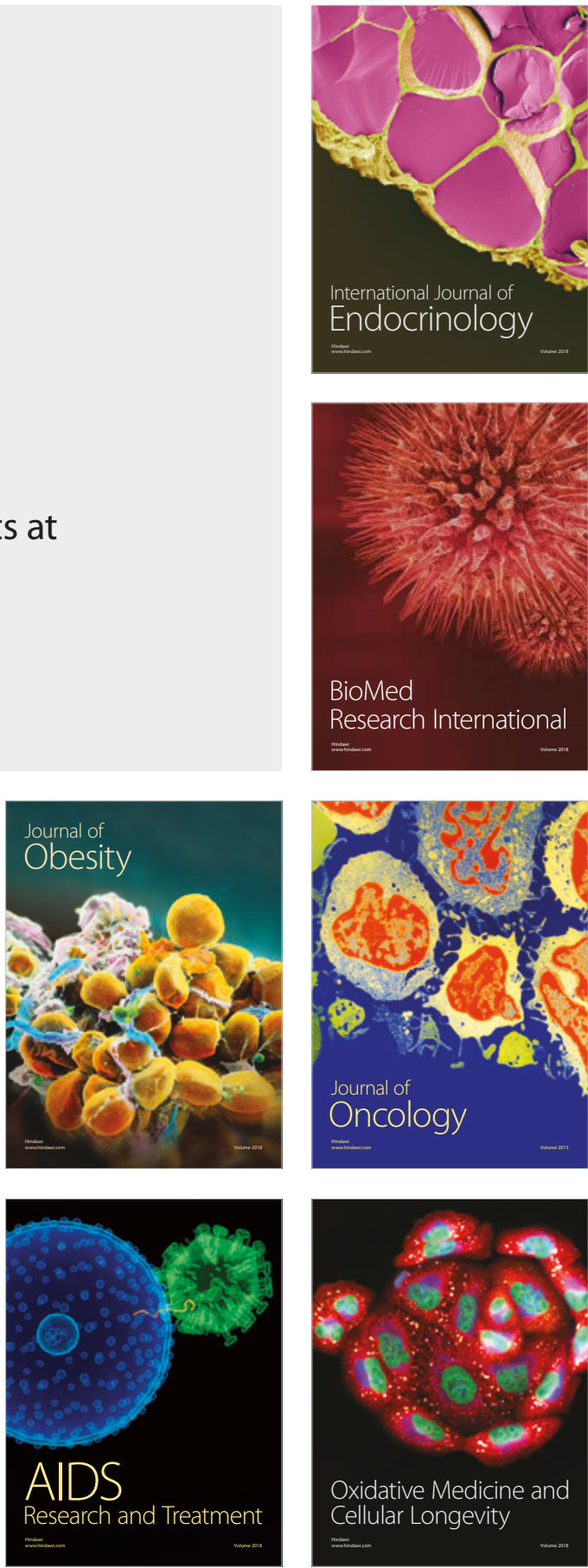